EON • Volume 14, Issue 5 - November 2021

\title{
Resources for Ethics in Data Publication
}

Kristen Overstreet ${ }^{1}$

${ }^{1}$ Managing Editor/Senior Partner, Origin Editorial, LLC

Published on: Nov 17, 2021

License: Creative Commons Attribution 4.0 International License (CC-BY 4.0). 


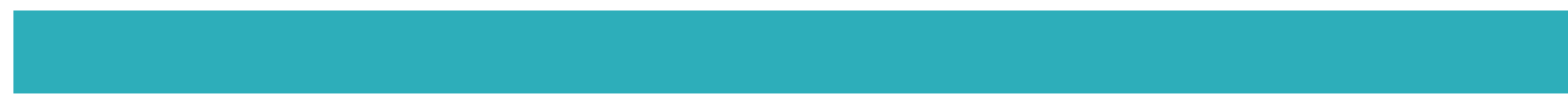

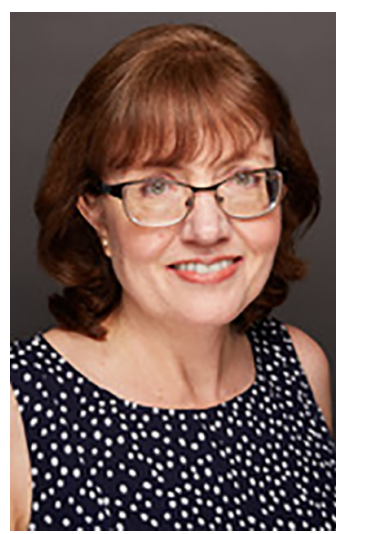

Kristen

Overstreet

Managing

Editor/Senior

Partner

Origin Editorial,

LLC
In an ideal world, all authors would deposit their study data in a repository and include the DOI(s) in their submitted manuscripts, and Editors and reviewers would have the time to access the data and verify the findings as part of their peer review. There are many obstacles to achieving this ideal, but the FORCE11 Research Data Publication Ethics Working Group, in collaboration with the Committee on Publication Ethics (COPE), is helping us to hurdle ethical issues related to data publication. The Working Group has published recommendations for handling ethical concerns that may arise in four key categories: Authorship \& Contribution Conflicts, Legal \& Regulatory Restrictions, Rigor, and Risk. You can access these recommendations here and read more about the development of the recommendations in this blog post.

Data sharing is a topic many of us may be just starting to think about in the editorial office -- whether to encourage or require data sharing, the policies and workflows needed, how to get all the stakeholders on board, and who is responsible for ensuring compliance with the policies; but thankfully, we now have these FORCE11 resources available to us to handle the ethical issues we will inevitably encounter. In addition to the already published resources, flowcharts showing the steps for managing an ethical issue are being developed for the four areas listed above.

\section{Case Examples from the Resources}

Authorship \& Contribution Conflicts: A postdoc works on a study, collecting and analyzing data for several years, and then takes a new job at another university. When reading a published article by his colleagues from this study, he realizes he has not been included in the author list for the article -- appropriately since he was not there to help with the writing -- but he also sees he has not been listed as an author on the submitted dataset that is cited in the article, and he should have been. He reaches out to the editorial office of the journal that published the article. What do you do? The editorial office should direct the author to submit his concern to the repository where the data were published. If the authorship metadata is updated in the repository and the authorship was published in the article's reference list, a correction could be 
published. If the authorship is not listed, no corrections may be needed. Future readers will see the corrected author list when they link to the dataset from the DOI.

Legal \& Regulatory Restrictions: A representative from a hospital contacts the editorial office to request retraction of an article reporting on data collected from the hospital records that fall outside the institution's regulations for data collection. What do you do? First, the journal will want to seek legal counsel from the journal owner's legal resources. Based on that counsel, the journal may want to contact the authors and request documentation of permissions obtained from the hospital to collect and share the data. The next steps depend upon the author's response and any documentation they provide. See next steps in the bulleted text under the "What actions should be taken for a published dataset? Who needs to be involved in this decision?" in the resource (page 6). The journal will need to consider how the issue affects the published article and may need to publish a correction, noting changes that were made to the dataset to come into compliance with the institution's regulations or consider retracting the article if regulations were breached.

Rigor: A reviewer alerts the editor of a journal that the data provided for review is incomplete and affects the findings reported in the manuscript. What should you do? The journal should halt the peer review process, contact the corresponding author, and ask for a response to the reviewer's concern. If the omission was an honest error and the author can revise and redeposit the dataset, the reviewers can be informed, and the peer review process can move forward. If the author cannot correct the issue, they should withdraw the manuscript from consideration. If the editor suspects misconduct, they should consult the COPE flowchart for handling concerns about data integrity in a submitted manuscript.

Risk: An editor reviews a submission prior to starting the peer review process and notices that data provided in the results include information that could allow the human subjects to be identified, and the authors have deposited those data in a repository and provided the link to the dataset in the manuscript. What should you do? The editor should contact the corresponding author and ask for evidence that the subjects provided explicit consent to allow data sharing and what de-identification of the data is required. The editor should also ask for the institutional review board (IRB) approval documentation. The editor may ask the authors to revise the reporting of their results to de-identify the data even if the subject provided permission and the study received IRB approval. The editor/editorial office should also alert the data repository. 
Many more case examples are included in the resources as well as recommendations for how to manage them. One step that is universal is to consider whether other parties might be affected and to take reasonable steps to notify them. Take a minute to familiarize yourself with these resources so you know where they are and what they offer when an ethical issue involving a paper's underlying data arises at your journal, and stay tuned for further updates from the Working Group on the publication of future resources, such as the flowcharts.

\section{References}

Puebla, I., Lowenberg, D., \& FORCE11 Research Data Publishing Ethics WorkGroup. (2021, September 6). Joint FORCE11 \& COPE Research Data Publishing Ethics Working Group Recommendations. DOI 10.5281/zenodo.5391293.

Puebla, I., Lowenberg, D. \& FORCE11 Research Data Publishing Ethics WorkGroup. (2021, September 7). Recommendations for the handling of ethical concerns relating to the publication of research data. Available at:

https://www.force11.org/article/recommendations-handling-ethical-concerns-relatingpublication-research-data 